\title{
The WTX/AMER1 gene family: evolution, signature and function
}

\author{
Agnès Boutet ${ }^{1,2}$, Glenda Comai ${ }^{1,2}$, Andreas Schedl $^{1,2^{*}}$
}

\begin{abstract}
Background: WTX is a novel gene mutated in a proportion of Wilms' tumors and in patients suffering from sclerosing bone dysplasia. On the molecular level WTX has been shown to act as an antagonist of canonical Wnt/ $\beta$ catenin signaling in fish and mammals thus linking it to an essential pathway involved in normal development and cancer formation. Interestingly, WTX seems to also localize to an intranuclear component called paraspeckles. In spite of the growing interest of molecular biologists in WTX, little is known about its paralogs and its phylogenetic history.

Results: Using the amino-acid sequence of WTX/AMER1 as a tool for the assignment of orthology and paralogy, we here identify two novel proteins, AMER2 and AMER3, as "WTX" related. This Amer gene family is present in all currently available vertebrate genome sequences, but not invertebrate genomes and is characterized by six conserved blocks of sequences. The phylogenetic analysis suggests that the protoAmer gene originated early in the vertebrate lineage and was then duplicated due to whole genome duplications (WGD) giving rise to the three different Amer genes.

Conclusion: Our study represents the first phylogenetic analysis of Amer genes and reveals a new vertebrate specific gene family that is likely to have played an important role in the evolution of this subphylum. Divergent and conserved molecular functions of Wtx/Amer1, Amer2 and Amer3 are discussed.
\end{abstract}

\section{Background}

Early 2007, a search for genes deleted in Wilms' tumors, a pediatric solid tumor of the kidney led to the identification of the X-linked gene WTX (also called AMER1) [1]. Using large-scale interactome mapping a second independent study demonstrated that WTX induces degradation of $\beta$-catenin via the proteasome system, thus identifying this gene as an important modulator of this crucial signaling pathway [2]. Wtx/Amer1 also physically interacts with APC [3], a tumor suppressor gene involved in colorectal cancer [4]. In addition, on the cellular level WTX localizes to subnuclear domains that have been identified as paraspeckles [5]. Recent analysis suggests that WTX may also play an important function during normal development: expression analysis demonstrated a dynamic expression pattern throughout embryogenesis [6] and mutations have been identified in patients suffering from a range of developmental defects including osteopathia striata congenita with cranial

\footnotetext{
* Correspondence: schedl@unice.fr

${ }^{1}$ INSERM, U636, F-06108 Nice, France

Full list of author information is available at the end of the article
}

sclerosis (OSCS) and cardiac anomalies [7]. To better characterize the functional and structural properties of the WTX/AMER1 gene it is essential to understand its molecular evolution and its phylogenetic history. Duplications are common events during evolution and are one of the main driving forces for the emergence of new genes that can lead to the appearance of new gene families. The aim of the present study was to identify potential new members of the "WTX/AMER" family, characterize their phylogenetic relationships and analyze their evolutionary history.

\section{Results and Discussion}

Wtx/Amer1 is the founding member of a novel vertebrate gene family

Using the WTX/AMER1 sequence as a bait for proteinprotein comparisons in the human genome, FAM123A (AMER2) and FAM123C (AMER3) were identified as two genes that share several domains of significant sequence identity with WTX/AMER1 and are located in chromosomes 13 and 2 respectively. Amer 2 and Amer 3 were also present in mouse mapping to chromosomes
C Biomed Central 
14 and 1 respectively. Alignment of the mouse and human sequences highlighted the presence of six highly conserved blocks that we named B1 to B6 (Figure 1 and additional file 1, Figure SM1). The mouse and human AMER proteins are encoded by a single exon although 5 ' untranslated sequences map to additional exons (additional file 1, Figure SM1). In the case of WTX/ AMER1 additional splicing within the coding exon can result in alternative protein variants [7]. These spliced forms are detected after transient transfection of mouse Wtx/Amer1 in human cells, but not in mouse embryos from E9.5 to E14.5 dpc (Figure 2). Sequencing of RTPCR products from the embryonic samples indicated the presence of a shorter transcript than expected for Amer2 (Figure 2). This result showed that splicing leads to an in-frame deletion of amino acids within the
$\mathrm{N}$-terminal region of Amer2. The existence of two variants for human AMER2 had already been reported by Grohmann et al. (2007) [3] after in silico analysis. Alignment of the spliced form of Amer2 with the full-length transcript revealed a splice site donor with an AG-GT core sequence which is universally found at the exon/ intron junction. Sequence analysis localized this splice site donor just after the conserved domain B2 (see Figure 1). By contrast, Amer3 does not seem to undergo splicing, as we detected only one long transcript by RT-PCR both in mouse embryos and in transiently transfected cells with mouse Amer3 (Figure 2).

To further investigate the evolutionary origin and phylogenetic distribution of the Amer genes, we extended the in silico searches to other available genomic databases. We could identify orthologs of Wtx/Amer1,

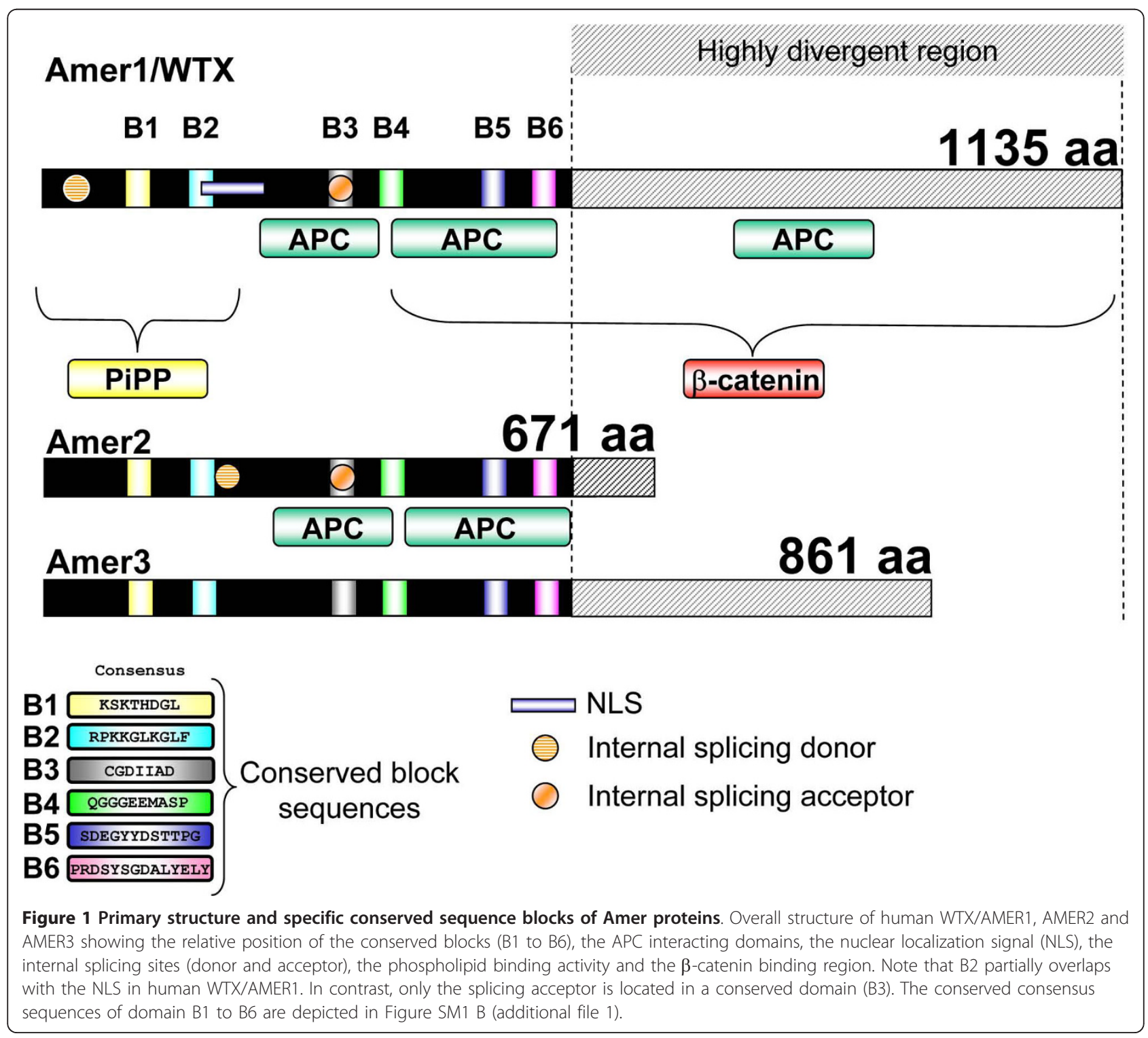


Amer 2 and Amer3 in primates (i.e. Pan troglodyte), and all other studied mammals (i.e. Canis familiaris), including the most basal therian groups, xenarthra (i.e. Dasypus novemcinctus), marsupials (i.e. Monodelphis domestica) and monotremes (i.e. Ornithorhynchus anatinus). The three Amer genes were also present in other tetrapod species such as birds (i.e. Gallus gallus), amphibians (i.e. Xenopus tropicalis) and in actinopterygians (i. e. the teleost fish Danio rerio) (for a complete list of orthologs see additional file 2; Table S1). We were unable to find any ortholog in cyclostome genomes such as the lamprey (Petromyzon marinus), but this is probably due to the fact that this genome has not yet been completely sequenced and assembled.

In stark contrast to their presence in apparently all vertebrates, no Amer genes could be identified in the genomes of model invertebrates including flies or nematodes and other protostomes such as mollusks (Lottia gigantea) and annelids (Capitella capitata). A similar situation already exists for other Wnt signaling components such as the $D k k$ family, which was believed to be vertebrate specific [8]. More recently, however, $D k k$ genes have been described in the diploblastic hydra and sea anemone [9]. It has, therefore, been postulated that gene families are missing in model protostomes due to the fast evolutionary rates and rampant gene loss in Drosophila and Caenorhabditis elegans genomes [10]. To test whether a similar situation may exist for the Amer gene family, we searched for Amer orthologs in a wide range of metazoans, from the most basal (cnidarians and placozoans) to the vertebrates' closest relatives. No hits in any of the analyzed genomes could be detected, not even in non-vertebrate chordates (i.e.
Branchiostoma floridae, Ciona intestinalis) and other non-vertebrate deuterostomes such as sea urchin (Strongylocentrotus purpuratus) despite the low occurrence of gene loss in some of these species [11]. Although we cannot rule out the possibility that Amerrelated genes are present in some of these species, but have diverged beyond recognition, our data strongly suggest that this gene family is vertebrate specific. While the six conserved blocks of sequences found in the human and mouse proteins are present in all the vertebrate orthologs (Figure 1 and additional file 1, Figure SM1), PROSITE analysis did not identify protein domains of known function (additional file 1, Figure SM2). Taken together, our data support the common origin of the three genes, which seemed to have arisen at the time of the origin of vertebrates.

\section{Phylogenetic relationship of Wtx/Amer1, Amer2 and Amer3}

To analyze in more detail the evolutionary history of the Amer gene family we constructed a phylogenetic tree using the protein sequences identified above. Phylogenetic analysis revealed that Wtx/Amer 1 , Amer 2 and Amer3 form three independent groups in each class of vertebrate species from fish to placental mammals (Figure 3A). Among the three genes Wtx/Amer 1 and Amer 2 show a higher extent of sequence similarity (see similarity percentage Figure 3B). In all the species analyzed, the three genes are located on three different chromosomes suggesting that they evolved by genome duplications rather than tandem duplication events (additional file 1, Figure SM3). If Wtx/Amer1, Amer2 and Amer 3 genes arise from a common ancestor, one 


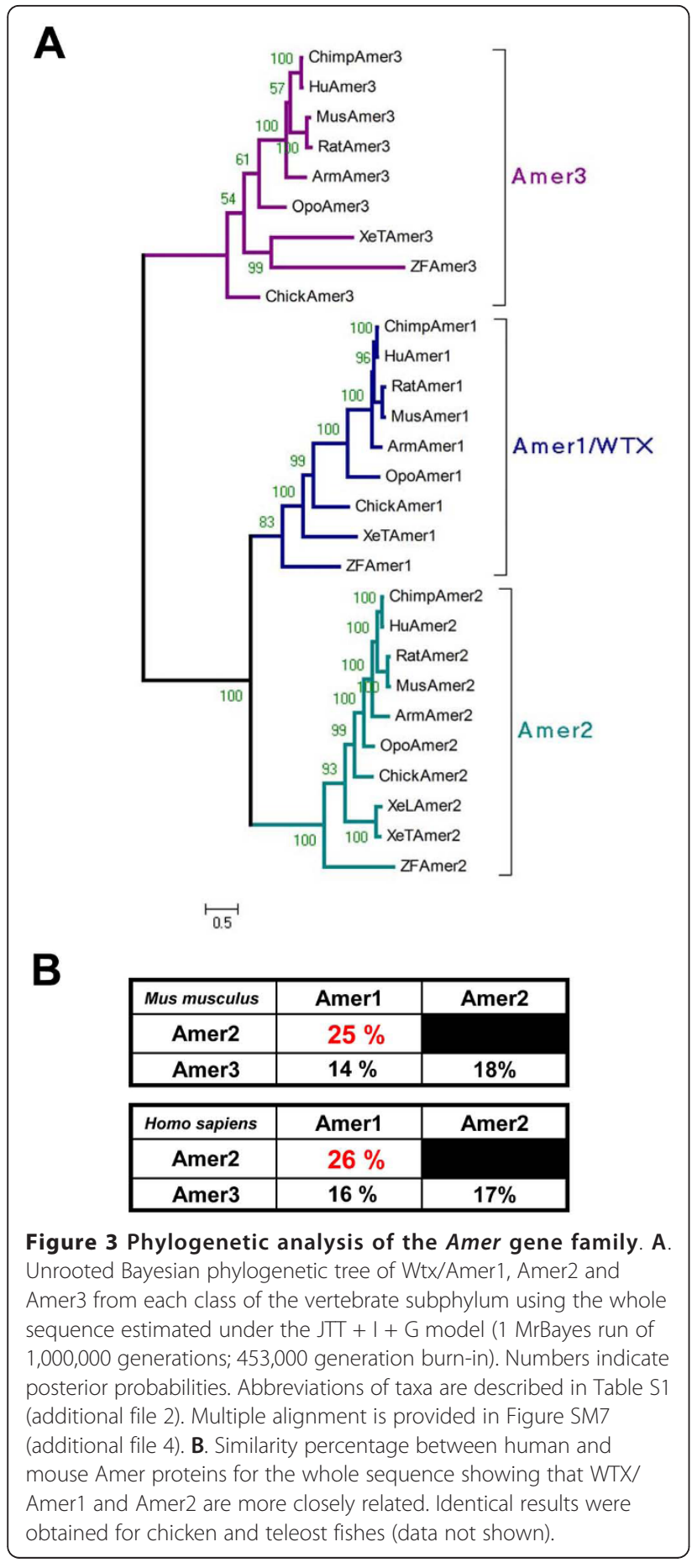

should expect to find them in the vicinity of a common gene family. Schematic representation of chromosomal locations for each gene is given in Figure SM3 (additional file 1). The following genes were found in syntenic position with Wtx/Amer1, Amer2 and Amer3: MTMR6/8 (myotubulin related protein 6/8) and $A R H$ GEF4/9 (cdc42 guanine nucleotide exchange factor (GEF) 4/9). In Drosophila the ortholog for both
MTMR6 and MTMR8, is the gene (CG3530) located on chromosome 2R. Similarly, the ortholog for ARHGEF4 and ARHGEF9 is RhoGEF3, a gene located on the Drosophila chromosome 3L (additional file 1, Figure SM3). At those two putative syntenic regions, we could not find any gene with significant level of sequence conservation to the Amer gene family. More precisely none of the six conserved sequence blocks could be found in the genes neighbouring CG3530 or RhoGEF3. The fact that ARHGEF4/9 is a neighbour of both Wtx/Amer1 and Amer3 and that MTMR6/8 neighbours both Wtx/Amer1 and Amer2 suggested that the ancestors of these two gene families (ARHGEF and MTMR) were located close to each other early in the vertebrate lineage (additional file 1, Figure SM4). This suggests a loss of paralogs after or between the two rounds of complete duplication in vertebrates (additional file 1, Figure SM4). Based on our analysis, we propose an evolutionary model (additional file 1, Figure SM4) that is consistent with the two rounds of complete genome duplication in vertebrates [12]. In our model, a putative Amer4 gene may have been lost after duplication and the presence of a "protoAmer" at the basis of the vertebrate lineage would represent the ancestral vertebrate condition preceding the gene split into Amer1/2 and Amer3/4 (additional file 1, Figure SM4). Surprisingly, the ortholog distribution of Wtx/Amer1, Amer 2 and Amer3 in teleosts does not reflect the additional round of genome duplication that occurred in these species (Figure 3A). A secondary loss of the duplicated genes can explain the present situation. Analysis of the degree of identical residues between human and zebrafish Amer gene products shows that it is relatively conserved (additional file 3, Figure SM5). In addition, residues showing a high degree of similarity after multiple alignment of Amer proteins from human and zebrafish correspond to the specific conserved domains found in Amer proteins (Block B1 to B6, Figure 1; additional file 1, Figure SM1).

The radial phylogenetic tree (additional file 3, Figure SM6) confirmed the subdivision of the three genes into three different groups.

\section{Divergent and conserved molecular functions of Wtx/Amer1 genes}

As noted by François Jacob in 1977, it is at the molecular level that the tinkering aspect of evolution becomes most apparent [13]. Even when gene families evolve by duplication, their members can undergo significant changes leading to structural and functional diversity. According to this view, the protoAmer gene may have encoded a short protein to which the highly divergent C-terminal half of WTX/AMER1 and AMER3 might have been added at a later time point during evolution (Figure 1). This C-terminal part of the WTX/AMER1 
protein is of importance as it has been demonstrated to be involved in the degradation of the Wnt signaling pathway effector $\beta$-catenin [2]. According to the primary structure we expect that Amer2 and Amer3 are unable to inhibit $\beta$-catenin activity. Indeed, Amer 2 failed to counteract $\beta$-catenin activation in an in vitro reporter system (Figure 4). While Wtx/Amer1 and Amer3 are both long proteins, their $\mathrm{C}$ terminal half lacks significant sequence identity and our phylogenetic analysis places the two genes into two different groups (see radial phylogenetic tree and cladogramme). Functional experiments revealed that Amer3 was also unable to decrease $\beta$-catenin dependent gene activation (Figure 4). Taken together these data indicate that Wtx/Amer1 might have been co-opted to interact with $\beta$-catenin. While addition of the $\beta$-catenin interaction domain to Wtx/Amer 1 seems to be the most likely scenario, we cannot completely rule out the possibility of a functional loss of this capability in Amer 2 and 3 proteins. In contrast, the function of the Amer gene family as proteins that recruit APC to the membrane might be conserved, as both WTX/AMER1 and AMER2 possess sequences that allow the molecular interaction with APC (Figure 1; [3]). The APC interacting domains overlap four of the six conserved blocks (Figure 1) indicating that AMER3 might also be involved in APC shuttling. If so, it seems that the ability to recruit APC is one of the ancestral functions of the Amer family.

\section{Conclusion}

In this study we reported the first phylogenetic analysis of the Amer gene family and identify Amer3 as a novel family member. The common ancestry of the three Amer genes is supported by the presence of six conserved sequence blocks in all the orthologs, as well as the syntenic conservation with NTMR and ARHGEF paralogs in their three genomic loci. The absence of any sequence similarity to Amer genes in invertebrate genomes and the presence of three Amer paralogs in all studied vertebrate species strongly support the hypothesis that these genes are novel inventions that originated early in vertebrate evolution. Additional functional studies using animal models misexpressing Amer 2 and Amer 3 or knock out alleles will be required to determine their exact function in embryogenesis. Given the importance of Wtx/Amer1 in normal development and its involvement in human pathologies, it is tempting to speculate that mutations in Amer2 and Amer3 may also underlie developmental disorders.

\section{Methods}

\section{Database search and sequence queries}

Search for Amer homologous proteins in vertebrates was made through the NCBI and Ensembl (http://www. ensembl.org; release 56, september 2009) protein databases (see the complete list in additional file 2; Table S1). In silico proteins were obtained using GenomeScan software (http://genes.mit.edu/genomescan.html). In order to find potential orthologs in invertebrate

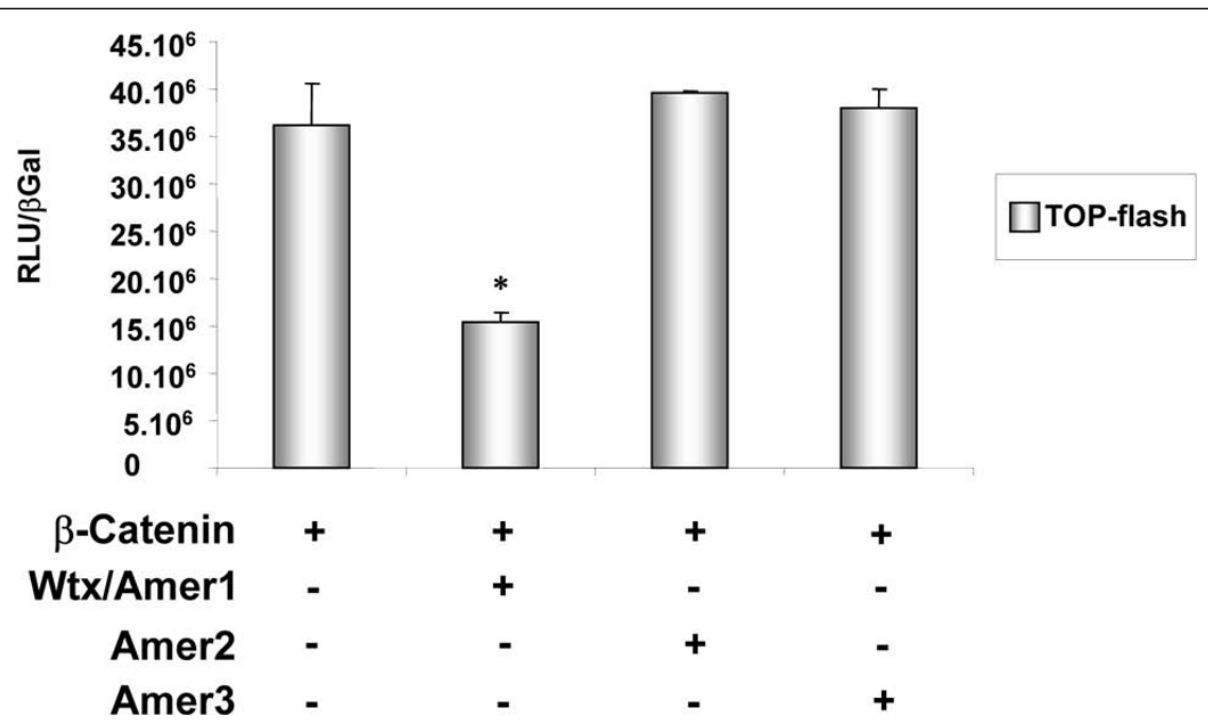

Figure 4 Regulation of $\beta$-catenin activity by Amer proteins. Co-transfection experiments of $\beta$-catenin and mouse Amer expression constructs together with a luciferase reporter gene (TOP-flash) into HEK293T cells are shown. The TOP-FLASH construct is designed to measure transcriptional activation mediated by $\beta$-catenin. As previously reported, WTX/AMER1 acts as a negative regulator of $\beta$-catenin activity. In contrast, AMER2 and AMER3 do not interfere with $\beta$-catenin dependent activation of the TOP-flash reporter gene. Luciferase values were normalized to $\beta$-galactosidase. Data are the average of two independent experiments \pm SEM. ${ }^{*} p \leq 0.01$. 
genomes, mouse Wtx/Amer1, Amer2 and Amer3 were blasted against the amphioxus (http://genome.jgi-psf. org/Brafl1/Brafl1.home.html), the sea urchin (http:// www.spbase.org/SpBase/) the sea anemone (Nematostella vectensis, http://genome.jgi-psf.org/Nemve1/ Nemve1.home.html), annelid (Lottia gigantea, http:// genome.jgi-psf.org/Lotgi1/Lotgi1.home.html), mollusk (Capitella capitata, http://genome.jgi-psf.org/Capca1/ Capca1.home.html) and placozoan (Trichoplax adhaerens, http://genome.jgi-psf.org/Triad1/Triad1.home.html) genomes using tblastn under unrestrictive conditions (e-value $=100)$. No hits were found. The absence of Wtx/Amer1 from the Drosophila genome has been previously reported [1].

\section{Alignment and phylogenetic analysis}

Amino acid sequences of Wtx/Amer1, Amer2, Amer3 were aligned using Clustal W [14] and manually corrected with BioEdit $[14,15]$. Bayesian Inference trees were performed using MrBayes 3.1.2 [16,17] with the model recommended by ProTest 1.4 [18] under the Akaike information and the Bayesian information criterions. We used the JTT $+\mathrm{I}+\mathrm{G}$ model for both trees. Convergence was reached when the value for the standard deviation of split frequencies stayed below 0.01 . Burn-in was determined by plotting parameters across all runs for both analysis: all trees prior to convergence were discarded and consensus trees were calculated for the remaining trees. We used one MrBayes run of 1,000,000 generations and 453,000 generation burn-in for the tree presented in Figure 3A and one MrBayes run of 2,000,000 generations and 1,893,000 generation burn-in for the tree presented in Figure SM6 (additional file 3 ). The trees were viewed and edited with the TreeExplorer programme in MEGA 4.0 [19]. Maximum likelihood analysis was conducted using RAxML version 7.0.3 [20] using a JTT model of evolution, 1000 bootstrap replicates and the rapid bootstrapping algorithm. The phylogenetic tree obtained using ML is consistent with the one obtained by Bayesian inference (data not shown). Alignment files used to calculate the phylogenetic trees are presented in Figure SM7 and SM8 (additional file 4 and 5 respectively).

\section{Plasmids}

The plasmid expressing human $\beta$-catenin was kindly provided by Jurgen Behrens (University of Erlangen, Germany). The TOP-FLASH and FOP-FLASH luciferase reporter constructs have been described previously [21]. Briefly, the TOP-FLASH construct is designed to measure transcriptional activation mediated by $\beta$-catenin and FOP-FLASH is the mutated counterpart of the
TOP-FLASH plasmid. Mouse Wtx/Amer1, Amer2 and Amer3 expression plasmids were obtained by PCR amplification of the coding sequence from BAC constructs (bMQ-277D5 for Wtx/Amer1, bMQ-344G22 for Amer2 and bMQ-123E19 for Amer3) (Geneservice, Cambridge, UK)) and insertion into the pAcGFP1-C1 plasmid (Clontech). All constructs were verified by DNA sequencing.

\section{Detection of Wtx/Amer1 and Amer2 variants by RT-PCR}

Total RNA from E9.5 to E14.5 dpc mouse embryos or Wtx/Amer1, Amer2 or Amer3 transfected HEK293T cells was extracted using TRIzol reagent (Invitrogen) and RNA purification was performed using the RNeasy Mini kit (Qiagen) and Rnase-free DNAse digestion (Qiagen). cDNA were synthesized from $1 \mu \mathrm{g}$ of RNA using the MMLV reverse transcriptase system and random hexamers (Invitrogen). All PCR assays were performed using the GoTaq ${ }^{\circ}$ Green Mix for PCR (Promega). Amplification of the $\mathrm{N}$-terminus of Wtx/ Amer1, Amer2 and Amer3 was performed using three different pairs of primers for each gene. Primer sequences used in Figure 2 are as follows: Wtx/Amer1, foward: tgaggcaacagaaggacca, reverse: tggagagtcaacaggatgaagctgttcaa; Amer2, foward: atggactcgcattgtgagtgcg, reverse: cgagctcccatctgcaaa; Amer3, foward: gaggagaggaaagaccttcatc, reverse: tcccagaacttgttgaagtctg. The other primer pairs used in this assay are available on demand. Briefly, all forward primers were located in the non-coding exon 1 or at the 5 ' end of exon 2 and all reverse primers just downstream of the internal splicing acceptor. PCR products were sequenced (SeqLAB, Sequence Laboratories, Göttingen, Germany) to check the specificity of Amer variants.

\section{Reporter assay}

HEK293T cells were cultured in DMEM supplemented with $10 \%(\mathrm{v} / \mathrm{v})$ fetal calf serum, in a $5 \% \mathrm{CO} 2$ humidified atmosphere. HEK293T cells were transiently transfected with the TOP-FLASH reporter or its mutated counterpart (FOP-FLASH) and $\beta$-catenin with either $W t x /$ Amer1, Amer 2 and Amer 3 expression plasmids (200 ng each). Cells were also co-transfected with $50 \mathrm{ng}$ of CMV (cytomegalovirus)- $\beta$-galactosidase as an internal control. Transient transfection of expression plasmids was performed with Fugene according to manufacturers instructions (Invitrogen). After $36 \mathrm{~h}$, luciferase activity was measured according to the Luciferase Assay System (Promega) and data were normalized to $\beta$-galactosidase activity and plotted. Luciferase activities obtained after transfection with the FOP-FLASH construct are not shown as they were close to zero for each condition. 


\section{Additional material}

Additional file 1: Additional figures (SM1-4) and corresponding captions. Figure SM1 is a figure showing the exon-intron structure of Amer genes and the consensus sequences of the six specific conserved domains found in all Amer proteins. Figure SM2 displays the protein domains for each Amer protein. Figure SM3 shows the conserved synteny of Amer genes in vertebrates. Figure SM4 proposes an evolutionary scenario for the origin of the Amer gene family.

Additional file 2: Table S1. List of Wtx/Amer1, Amer2 and Amer3 orthologs.

Additional file 3: Additional figures (SM5-6) and corresponding captions. Figure SM5 displays multiple alignment of Amer proteins from human and zebrafish. Figure SM6 shows the phylogenetic tree generated with a wide array of Amer proteins.

Additional file 4: Additional figure SM7. Figure SM7 contains multiple alignment performed with Wtx/Amer1, Amer2 and Amer3 from each class of the vertebrate subphylum using the whole sequence ( 28 sequences in total).

Additional file 5: Additional figure SM8. Figure SM8 contains multiple alignment performed with a broader array of Wtx/Amer1, Amer2 and Amer3 proteins (73 sequences in total).

\section{Abbreviations}

AMER: APC membrane recruitment; APC: adenomatous polyposis coli; WDG: whole duplication genome; NLS: nuclear localization signal; OSCS: osteopathia striata congenital with cranial sclerosis; DKK: Dickkopf.

\section{Acknowledgements}

We wish to acknowledge Ignacio Maeso for help with phylogenetic analysis and valuable comments concerning the manuscript. We also thank Michael Clarkson for continuous discussion in the lab. We are grateful to Jurgen Behrens (University of Erlangen, Germany) for the $\beta$-catenin plasmid and Laurent Ruel and Miguel Caetano Monteiro (CNRS UMR6543, Nice University, France) for providing the TOP-FLASH and FOP-FLASH plasmids. A.B. and G.C. are supported by ARC (French national council for cancer research) and a Marie-Curie fellowship as part of the InterDeC PhD program. The research in the laboratory is supported by grants from ARC (grant 1130) and the Association for International Cancer Research (AICR grant 09-0752).

\section{Author details}

'INSERM, U636, F-06108 Nice, France. 'Université de Nice-Sophia Antipolis, Laboratoire de génétique du développement Normal et Pathologique, F06108 Nice, France.

\section{Authors' contributions}

AS initially identified Amer2 and Amer3 from the database. GC contributed to the ortholog searches and performed the splicing analysis. AB carried out alignment, phylogenetic construction and functional experiments. $A S$ and $A B$ wrote the paper. All authors read and approved the final manuscript.

Received: 4 February 2010 Accepted: 15 September 2010 Published: 15 September 2010

\section{References}

1. Rivera MN, Kim WJ, Wells J, Driscoll DR, Brannigan BW, Han M, Kim JC, Feinberg AP, Gerald WL, Vargas SO, Chin L, lafrate AJ, Bell DW, Haber DA: An X chromosome gene, WTX, is commonly inactivated in Wilms tumor. Science 2007, 315:642-645.

2. Major MB, Camp ND, Berndt JD, Yi X, Goldenberg SJ, Hubbert C, Biechele TL, Gingras AC, Zheng N, Maccoss MJ, Angers S, Moon RT: Wilms tumor suppressor WTX negatively regulates WNT/beta-catenin signaling. Science 2007, 316:1043-1046.

3. Grohmann A, Tanneberger K, Alzner A, Schneikert J, Behrens J: AMER1 regulates the distribution of the tumor suppressor APC between microtubules and the plasma membrane. J Cell Sci 2007, 120:3738-3747.
4. Groden J, Thliveris A, Samowitz W, Carlson M, Gelbert L, Albertsen H, Joslyn G, Stevens J, Spirio L, Robertson M, Sargeant L, Krapcho K, Wolff E, Burt R, Hughes JP, Warrington J, McPherson J, Wasmuth J, Le Paslier D, Abderrahim H, Cohen D, Leppert M, Ray White: Identification and characterization of the familial adenomatous polyposis coli gene. Cell 1991, 66:589-600.

5. Rivera MN, Kim WJ, Wells J, Stone A, Burger A, Coffman EJ, Zhang J, Haber DA: The tumor suppressor WTX shuttles to the nucleus and modulates WT1 activity. Proc Natl Acad Sci USA 2009, 106:8338-43.

6. Comai G, Boutet A, Neirijnck Y, Schedl A: Expression patterns of the Wtx/ Amer gene family during mouse embryonic development. Dev Dyn 2010, 239:1867-78.

7. Jenkins ZA, van Kogelenberg M, Morgan T, Jeffs A, Fukuzawa R, Pearl E, Thaller C, Hing AV, Porteous ME, Garcia-Miñaur S, Bohring A, Lacombe D, Stewart F, Fiskerstrand T, Bindoff L, Berland S, Adès LC, Tchan M, David A, Wilson LC, Hennekam RC, Donnai D, Mansour S, Cormier-Daire V, Robertson SP: Germline mutations in WTX cause a sclerosing skeletal dysplasia but do not predispose to tumorigenesis. Nat Genet 2009, 41:95-100.

8. Yamaguchi TP: Heads or tails: Wnts and anterior-posterior patterning. Curr Biol 2001, 11:R713-R724

9. Guder C, Philipp I, Lengfeld T, Watanabe H, Hobmayer B, Holstein TW: The Wnt code: cnidarians signal the way. Oncogene 2006, 25:7450-7460.

10. Miller DJ, Ball EE, Technau U: Cnidarians and ancestral genetic complexity in the animal kingdom. Trends Genet 2005, 21:536-539.

11. Holland LZ, Albalat R, Azumi K, Benito-Gutiérrez E, Blow MJ, BronnerFraser M, Brunet F, Butts T, Candiani S, Dishaw LJ, Ferrier DE, GarciaFernàndez J, Gibson-Brown JJ, Gissi C, Godzik A, Hallböök F, Hirose D, Hosomichi K, Ikuta T, Inoko H, Kasahara M, Kasamatsu J, Kawashima T, Kimura A, Kobayashi M, Kozmik Z, Kubokawa K, Laudet V, Litman GW, McHardy AC, et al: The amphioxus genome illuminates vertebrate origins and cephalochordate biology. Genome Res 2008, 18:1100-1111.

12. Ohno S: Evolution by gene duplication. Springer, Berlin Heidelberg New York 1970.

13. Jacob F: Evolution and tinkering. Science 1977, 196:1161-1166.

14. Thompson JD, Higgins DG, Gibson TJ: CLUSTAL W: improving the sensitivity of progressive multiple sequence alignment through sequence weighting, position-specific gap penalties and weight matrix choice. Nucleic Acids Res 1994, 22:4673-4680.

15. Tippmann HF: Analysis for free: comparing programs for sequence analysis. Brief Bioinform 2004, 5:82-87.

16. Huelsenbeck JP, Ronquist F: MRBAYES: Bayesian inference of phylogenetic trees. Bioinformatics 2001, 17:754-755.

17. Ronquist F, Huelsenbeck JP: MrBayes 3: Bayesian phylogenetic inference under mixed models. Bioinformatics 2003, 19:1572-1574

18. Abascal F, Zardoya R, Posada D: ProtTest: selection of best-fit models of protein evolution. Bioinformatics 2005, 21:2104-2105.

19. Kumar S, Nei M, Dudley J, Tamura K: MEGA: a biologist-centric software for evolutionary analysis of DNA and protein sequences. Brief Bioinform 2008, 9:299-306

20. Stamatakis A: RAxML-VI-HPC Maximum Likelihood-based Phylogenetic Analyses with thousands of taxa and mixed models. Bioinformatics 2006, 22:2688-2690.

21. Korinek V, Barker N, Morin PJ, van Wichen D, de Weger R, Kinzler KW, Vogelstein B, Clevers $\mathrm{H}$ : Constitutive transcriptional activation by a betacatenin-Tcf complex in APC-/- colon carcinoma. Science 1997, 275:1784-1787.

doi:10.1186/1471-2148-10-280

Cite this article as: Boutet et al:: The WTX/AMER1 gene family: evolution, signature and function. BMC Evolutionary Biology 2010 10:280. 